W.Ogryczak: On Principles of Fair Resource Allocation for Importance Weighted Agents, in: Proceedings International Workshop on Social Informatics SOCINFO 2009, IEEE Computer Society Press, 2009, 57-62. 


\title{
On Principles of Fair Resource Allocation for Importance Weighted Agents
}

\author{
Włodzimierz Ogryczak \\ Warsaw University of Technology \\ Institute of Control \& Computation Engineering \\ 00-665 Warsaw, Poland \\ E-mail: ogryczak@ia.pw.edu.pl
}

\begin{abstract}
Resource allocation problems are concerned with the allocation of limited resources among competing activities so as to achieve the best performances. However, in systems which serve many users there is a need to respect some fairness rules while looking for the overall efficiency. The concepts of multiple criteria equitable optimization can effectively be used to generate various fair and efficient allocation schemes. In this paper we show how the importance weights allocated to several agents can be introduced into the fairness concepts and models.
\end{abstract}

\section{INTRODUCTION}

Resource allocation problems are concerned with the allocation of limited resources among competing activities. In this paper, we focus on approaches that, while allocating resources to maximize the system efficiency, they also attempt to provide a fair treatment of all the competing activities [10]. The problems of efficient and fair resource allocation arise in various systems which serve many users, like in telecommunication systems among others. In networking a central issue is how to allocate bandwidth to flows efficiently and fairly [1], [4], [20]. The issue of equity is widely recognized in location analysis of public services, where the agents of a system are entitled to fair treatment according to community regulations. In such problems, the decisions often concern the placement of a service center or another facility in a position so that the users are treated in an equitable way, relative to certain criteria. Moreover, uniform individual outcomes may be associated with some events rather than physical users, like in many dynamic optimization problems where uniform individual criteria represent a similar event in various periods and all they are equally important.

The generic resource allocation problem may be stated as follows. Each activity is measured by an individual performance function that depends on the corresponding resource level assigned to that activity. A larger function value is considered better, like the performance measured in terms of quality level, capacity, service amount available, etc. Models with an (aggregated) objective function that maximizes the mean (or simply the sum) of individual performances are widely used to formulate resource allocation problems, thus defining the so-called mean solution concept. This solution concept is primarily concerned with the overall system efficiency. As based on averaging, it often provides solution where some smaller services are discriminated in terms of allocated resources. An alternative approach depends on the so-called Max-Min solution concept, where the worst performance is maximized. The Max-Min approach is consistent with Rawlsian theory of justice [21], especially when additionally regularized with the lexicographic order. The latter is called the Max-Min Fairness (MMF) and commonly used in networking [20]. Allocating the resources to optimize the worst performances may cause, however, a large worsening of the overall (mean) performances.

Fairness is, essentially, an abstract socio-political concept that implies impartiality, justice and equity [21]. In order to ensure fairness in a system, all system entities have to be equally well provided with the system's services. This leads to concepts of fairness expressed by the equitable efficiency [8], [10]. The concept of equitably efficient solution is a specific refinement of the Pareto-optimality taking into account the inequality minimization according to the Pigou-Dalton approach.

The paper is organized as follows. In the next section the equitable optimization with the preference structure that complies with both the efficiency (Pareto-optimality) and with the Pigou-Dalton principle of transfers is used to formalize the fair solution concepts. Further the introduction of the agents weights into the fair dominance relation as well as into specific fair solution concepts is analyzed.

\section{EQUITY AND FAIRNESS}

\section{A. Fair Dominance}

The generic resource allocation problem may be stated as follows. There is a system dealing with a set $I$ of $m$ services. There is given a measure of services realization within a system. The measure usually expresses the service quality but may modeled in a more subjective way. There is also given a set $Q$ of allocation patterns (allocation decisions). For each service $i \in I$ a function $f_{i}(\mathbf{x})$ of the allocation pattern $\mathbf{x} \in Q$ has been defined. This function, called the individual objective function, measures the outcome (effect) $y_{i}=f_{i}(\mathbf{x})$ of allocation $\mathrm{x}$ pattern for service $i$. In typical formulations a larger value of the outcome means a better effect (higher service quality or agent satisfaction). Otherwise, the outcomes can be replaced with their complements to some large number. Therefore, without loss of generality, we can assume that each individual outcome $y_{i}$ is to be maximized which allows us 
to view the generic resource allocation problem as a vector maximization model:

$$
\max \{\mathbf{f}(\mathbf{x}): \mathbf{x} \in Q\}
$$

where $\mathbf{f}(\mathbf{x})$ is a vector-function that maps the decision space $X=R^{n}$ into the criterion space $Y=R^{m}$, and $Q \subset X$ denotes the feasible set.

Model (1) only specifies that we are interested in maximization of all objective functions $f_{i}$ for $i \in I=\{1,2, \ldots, m\}$. In order to make it operational, one needs to assume some solution concept specifying what it means to maximize multiple objective functions. The solution concepts may be defined by properties of the corresponding preference model. The preference model is completely characterized by the relation of weak preference, denoted hereafter with $\succeq$. Namely, the corresponding relations of strict preference $\succ$ and indifference $\cong$ are defined by the following formulas:

$$
\begin{aligned}
& \mathbf{y}^{\prime} \succ \mathbf{y}^{\prime \prime} \quad \Leftrightarrow \quad\left(\mathbf{y}^{\prime} \succeq \mathbf{y}^{\prime \prime} \quad \text { and } \quad \mathbf{y}^{\prime \prime} \nsucceq \mathbf{y}^{\prime}\right), \\
& \mathbf{y}^{\prime} \cong \mathbf{y}^{\prime \prime} \quad \Leftrightarrow \quad\left(\mathbf{y}^{\prime} \succeq \mathbf{y}^{\prime \prime} \quad \text { and } \quad \mathbf{y}^{\prime \prime} \succeq \mathbf{y}^{\prime}\right) .
\end{aligned}
$$

The standard preference model related to the Pareto-optimal (efficient) solution concept assumes that the preference relation $\succeq$ is reflexive:

$$
\mathbf{y} \succeq \mathbf{y}
$$

transitive:

$$
\left(\mathbf{y}^{\prime} \succeq \mathbf{y}^{\prime \prime} \quad \text { and } \quad \mathbf{y}^{\prime \prime} \succeq \mathbf{y}^{\prime \prime \prime}\right) \quad \Rightarrow \quad \mathbf{y}^{\prime} \succeq \mathbf{y}^{\prime \prime \prime},
$$

and strictly monotonic:

$$
\mathbf{y}+\varepsilon \mathbf{e}_{i} \succ \mathbf{y} \quad \text { for } \varepsilon>0 ; i=1, \ldots, m,
$$

where $\mathbf{e}_{i}$ denotes the $i$-th unit vector in the criterion space. The last assumption expresses that for each individual objective function more is better (maximization). The preference relations satisfying axioms (2)-(4) are called hereafter rational preference relations. The rational preference relations allow us to formalize the Pareto-optimality (efficiency) concept with the following definitions. We say that outcome vector $\mathbf{y}^{\prime}$ rationally dominates $\mathbf{y}^{\prime \prime}\left(\mathbf{y}^{\prime} \succ_{r} \mathbf{y}^{\prime \prime}\right)$, iff $\mathbf{y}^{\prime} \succ \mathbf{y}^{\prime \prime}$ for all rational preference relations $\succeq$. We say that feasible solution $\mathbf{x} \in Q$ is a Pareto-optimal (efficient) solution of the multiple criteria problem (1), iff $\mathbf{y}=\mathbf{f}(\mathbf{x})$ is rationally nondominated.

In order to ensure fairness in a system, all system entities have to be equally well provided with the system's services. This leads to concepts of fairness expressed by the equitable rational preferences [8]. First of all, the fairness requires impartiality of evaluation, thus focusing on the distribution of outcome values while ignoring their ordering. That means, in the multiple criteria problem (1) we are interested in a set of outcome values without taking into account which outcome is taking a specific value. Hence, we assume that the preference model is impartial (anonymous, symmetric):

$$
\left(y_{\pi(1)}, \ldots, y_{\pi(m)}\right) \cong\left(y_{1}, \ldots, y_{m}\right) \quad \forall \pi \in \Pi(I)
$$

where $\Pi(I)$ denotes the set of all permutations of $I$. The impartiality axiom (5) means that any permuted outcome vector is indifferent in terms of the preference relation. Further, fairness requires equitability of outcomes which causes that the preference model should satisfy the (Pigou-Dalton) principle of transfers. The principle of transfers states that a transfer of any small amount from an outcome to any other relatively worse-off outcome results in a more preferred outcome vector, i.e., whenever $y_{i^{\prime}}>y_{i^{\prime \prime}}$ then

$$
\mathbf{y}-\varepsilon \mathbf{e}_{i^{\prime}}+\varepsilon \mathbf{e}_{i^{\prime \prime}} \succ \mathbf{y} \text { for } 0<\varepsilon<\left(y_{i^{\prime}}-y_{i^{\prime \prime}}\right)
$$

The rational preference relations satisfying additionally axioms (5) and (6) are called hereafter fair (equitable) rational preference relations. We say that outcome vector $\mathbf{y}^{\prime}$ fairly (equitably) dominates $\mathbf{y}^{\prime \prime}\left(\mathbf{y}^{\prime} \succ_{e} \mathbf{y}^{\prime \prime}\right)$, iff $\mathbf{y}^{\prime} \succ \mathbf{y}^{\prime \prime}$ for all fair rational preference relations $\succeq$. In other words, $\mathbf{y}^{\prime}$ fairly dominates $\mathbf{y}^{\prime \prime}$, if there exists a finite sequence of vectors $\mathbf{y}^{j}$ $(j=1,2, \ldots, s)$ such that $\mathbf{y}^{1}=\mathbf{y}^{\prime \prime}, \mathbf{y}^{s}=\mathbf{y}^{\prime}$ and $\mathbf{y}^{j}$ is constructed from $y^{j-1}$ by application of either permutation of coordinates, equitable transfer, or increase of a coordinate. An allocation pattern $\mathrm{x} \in Q$ is called fairly (equitably) efficient or simply fair if $\mathbf{y}=\mathbf{f}(\mathbf{x})$ is fairly nondominated. Note that each fairly efficient solution is also Pareto-optimal, but not vice verse.

Example 1: Let us consider a simple resource allocation problem with three agents and three potential allocations. The potential resource allocations schemes generate the outcome vectors $\mathbf{y}^{\prime}=(5,3,1), \mathbf{y}^{\prime \prime}=(3,3,3)$ and $\mathbf{y}^{\prime \prime \prime}=(4,5,9)$, respectively. Note that the perfectly equal outcome vector $\mathbf{y}^{\prime \prime}$ with all the outcomes 3 fairly dominates $\mathbf{y}^{\prime}$ since $\mathbf{y}^{\prime \prime}=$ $(3,3,3)$ can be obtained from $\mathbf{y}^{\prime}=(5,3,1)$ by application of one equitable transfer. On the other hand, outcome vector $\mathbf{y}^{\prime \prime}$ is obviously worse than unequal vector $\mathbf{y}^{\prime \prime \prime}$ with all the outcomes greater than 3. Actually, the perfectly equal outcome vector $\mathbf{y}^{\prime \prime}$ is fairly dominated by unequal vector $\mathbf{y}^{\prime \prime \prime}$ since $\mathbf{y}^{\prime \prime \prime}=$ $(4,5,9) \geq(3,3,3)=\mathbf{y}^{\prime \prime}$. One may also notice that despite $\mathbf{y}^{\prime}$ is not Pareto dominated by $\mathbf{y}^{\prime \prime \prime}$, it is fairly dominated since vector $\mathbf{y}^{\prime \prime \prime}$ appropriately rearranged (permuted) to $(9,5,4)$ gets all the outcomes greater than the corresponding outcomes of $\mathbf{y}^{\prime}=(5,3,1)$.

The relation of equitable dominance $\succeq_{e}$ can be expressed as a vector inequality on the cumulative ordered outcomes. For the unweighted problem this can be mathematically formalized as follows. First, we introduce the ordering map $\Theta: R^{m} \rightarrow$ $R^{m}$ such that $\Theta(\mathbf{y})=\left(\theta_{1}(\mathbf{y}), \theta_{2}(\mathbf{y}), \ldots, \theta_{m}(\mathbf{y})\right)$, where $\theta_{1}(\mathbf{y}) \leq \theta_{2}(\mathbf{y}) \leq \cdots \leq \theta_{m}(\mathbf{y})$ and there exists a permutation $\tau$ of set $I$ such that $\theta_{i}(\mathbf{y})=y_{\tau(i)}$ for $i=1, \ldots, m$. This allows us to focus on distributions of outcomes impartially. Next, we apply cumulation to the ordered outcome vectors to get quantities

$$
\bar{\theta}_{i}(\mathbf{y})=\sum_{j=1}^{i} \theta_{j}(\mathbf{y}) \quad \text { for } i=1, \ldots, m .
$$

expressing, respectively, the worst outcome, the total of the two worst outcomes, the total of the three worst outcomes, etc. Pointwise comparison of the cumulated ordered outcomes $\bar{\Theta}(\mathbf{y})$ for vectors with equal means was extensively analyzed 
within the theory of equity [26] or the mathematical theory of majorization [11], where it is called the relation of Lorenz dominance or weak majorization, respectively. It includes the classical results allowing to express an improvement in terms of the Lorenz dominance as a finite sequence of PigouDalton equitable transfers. It can be generalized to vectors with various means, which allows one to justify the following statement [8], [13]. Outcome vector $\mathbf{y}^{\prime} \in Y$ fairly dominates $\mathbf{y}^{\prime \prime} \in Y$, if and only if $\bar{\theta}_{i}\left(\mathbf{y}^{\prime}\right) \geq \bar{\theta}_{i}\left(\mathbf{y}^{\prime \prime}\right)$ for all $i \in I$ where at least one strict inequality holds.

\section{B. Fair Solution Concepts}

Simple solution concepts for multiple criteria problems are defined by aggregation (or utility) functions $g: Y \rightarrow R$ to be maximized. Thus the multiple criteria problem (1) is replaced with the maximization problem

$$
\max \{g(\mathbf{f}(\mathbf{x})): \mathbf{x} \in Q\}
$$

In order to guarantee the consistency of the aggregated problem (8) with the maximization of all individual objective functions in the original multiple criteria problem (or Paretooptimality of the solution), the aggregation function must be strictly increasing with respect to every coordinate. In order to guarantee fairness of the solution concept (8), the aggregation function must be additionally symmetric (impartial) as well as be equitable (to satisfy the principle of transfers), i.e.,

$$
g\left(y_{1}, \ldots, y_{i^{\prime}}-\varepsilon, \ldots, y_{i^{\prime \prime}}+\varepsilon, \ldots, y_{m}\right)>g\left(y_{1}, \ldots, y_{m}\right)
$$

for any $0<\varepsilon<\left(y_{i^{\prime}}-y_{i^{\prime \prime}}\right)$. In the case of a strictly increasing symmetric function satisfying the requirement (9), we call the corresponding problem (8) a fair (equitable) aggregation of problem (1). Every optimal solution to the fair aggregation (8) of a multiple criteria problem (1) defines some fair (equitable) solution.

The simplest aggregation functions commonly used for the multiple criteria problem (1) are defined as the mean (average) outcome

$$
\mu(\mathbf{y})=\frac{1}{m} \sum_{i=1}^{m} y_{i}
$$

or the worst outcome

$$
M(\mathbf{y})=\min _{i=1, \ldots, m} y_{i} .
$$

The mean (10) is a strictly increasing function while the minimum (11) is only nondecreasing. Both the simplest aggregation functions, the sum (10) and the minimum (11), are symmetric although they do not satisfy the equitability requirement (9). To guarantee the fairness of solutions, some enforcement of concave properties is required. For any strictly concave, increasing utility function $u: R \rightarrow R$, the function $g(\mathbf{y})=\sum_{i=1}^{m} u\left(y_{i}\right)$ is a strictly monotonic and equitable thus defining a family of the fair aggregations

$$
\max \left\{\sum_{i=1}^{m} u\left(f_{i}(\mathbf{x})\right): \mathbf{x} \in Q\right\}
$$

Various concave utility functions $u$ can be used to define the fair aggregations (12) and the resulting fair solution concepts. In the case of the outcomes restricted to positive values, one may use logarithmic function thus resulting in the Proportional Fairness (PF) solution concept [7]. Actually, it corresponds to the so-called Nash criterion which maximizes the product of additional utilities compared to the status quo. Various other concave functions $u$ can be used to define fair aggregations and the resulting resource allocation schemes [9], [12].

The Max-Min solution may be regularized according to the Rawlsian principle of justice, leading to the lexicographic Max-Min concepts or the so-called Max-Min Fairness [6], [2] Actually, a wider class of fair solutions to problem (1) can be expressed as Pareto-optimal solutions for the multiple criteria problem with objectives $\bar{\Theta}(\mathbf{f}(\mathbf{x}))$

$$
\max \left\{\left(\bar{\theta}_{1}(\mathbf{f}(\mathbf{x})), \bar{\theta}_{2}(\mathbf{f}(\mathbf{x})), \ldots, \bar{\theta}_{m}(\mathbf{f}(\mathbf{x}))\right): \mathbf{x} \in Q\right\}
$$

The aggregation maximizing the sum of outcomes, corresponds to maximization of the last objective $\left(\bar{\theta}_{m}(\mathbf{f}(\mathbf{x}))\right)$ in problem (13). Similar, the Max-Min corresponds to maximization of the first objective $\left(\bar{\theta}_{1}(\mathbf{f}(\mathbf{x}))\right)$. For modeling various fair preferences one may use some combinations the criteria. In particular, for the weighted sum aggregation on gets $\sum_{i=1}^{m} s_{i} \bar{\theta}_{i}(\mathbf{y})$, which can be expressed in the form with weights $w_{i}=\sum_{j=i}^{m} s_{j}(i=1, \ldots, m)$ allocated to coordinates of the ordered outcome vector, i.e., as the so-called Ordered Weighted Average (OWA) [25]:

$$
\max \left\{\sum_{i=1}^{m} w_{i} \theta_{i}(\mathbf{f}(\mathbf{x})): \mathbf{x} \in Q\right\}
$$

If weights $w_{i}$ are strictly decreasing and positive, i.e. $w_{1}>$ $w_{2}>\cdots>w_{m-1}>w_{m}>0$, then each optimal solution of the OWA problem (14) is a fair solution of (1). The recent progress in optimization methods for ordered averages allows one to implement the OWA optimization quite effectively as an extension of the original constraints and criteria with simple linear inequalities [17].

\section{FAIRNESS With IMPORTANCE WeIGHTS}

\section{A. Importance Weighted Agents}

Frequently, one may be interested in putting into allocation models some additional agent weights $v_{i}>0$. Typically the model of distribution weights is introduced to represent the agent importance thus defining distribution of outcomes $y_{i}=f_{i}(\mathbf{x})$ according to measures defined by the weights $v_{i}$ for $i=1, \ldots, m$. Note that such distribution weights allow us for a clear interpretation of weights as the agent repetitions [3]. Splitting an agent into two agents does not cause any change of the final distribution of outcomes. For theoretical considerations one may assume that the problem is transformed (disaggregated) to the unweighted one (that means all the agent weights are equal to 1). Note that such a disaggregation is possible for integer as well as rational agent weights, but it usually dramatically increases the problem size. Therefore, we are interested in solution concepts which can be applied directly to the weighted problem. 
As mentioned, for some theoretical considerations it might be convenient to disaggregate the weighted problems into the unweighted one. Therefore, to simplify the analysis we will assume integer weights $v_{i}$, although while discussing solution concepts we will use the normalized agent weights $\bar{v}_{i}=v_{i} / \sum_{i=1}^{m} v_{i}$ for $i=1, \ldots, m$, rather than the original quantities $v_{i}$. Note that, in the case of unweighted problem (all $v_{i}=1$ ), all the normalized weights are given as $\bar{v}_{i}=1 / \mathrm{m}$. Furthermore, to avoid possible misunderstandings between the weighted outcomes and the corresponding unweighted form of outcomes we will use the following notation. Index set $I$ will always denote unweighted agents (with possible repetitions if originally weighted) and vector $\mathbf{y}=\left(y_{i}\right)_{i \in I}=$ $\left(y_{1}, y_{2}, \ldots, y_{m}\right)$ will denote the unweighted outcomes. While directly dealing with the weighted problem (without its disaggregation to the unweighted one) we will use $I_{v}$ to denote the set of agents and the corresponding outcomes will be represented by vector $\mathbf{y}=\left(y_{v_{i}}\right)_{i \in I_{v}}$. We illustrate this with the following small example.

Example 2: Let us consider a weighted resource allocation problem with two agents $\mathrm{C} 1$ and $\mathrm{C} 2$ having assigned demand weights $v_{1}=1$ and $v_{2}=9$, respectively. Their outcomes relate to two potential resource allocation decisions $\mathrm{A} 1$ and $\mathrm{A} 2$ are given as follows:

\begin{tabular}{r|rr} 
& $\mathrm{C} 1$ & $\mathrm{C} 2$ \\
\hline $\mathrm{A} 1$ & 10 & 0 \\
$\mathrm{~A} 2$ & 0 & 0 \\
\hline
\end{tabular}

Hence, $I_{v}=\{1,2\}$ and the potential resource allocations generate two outcome vectors $\mathbf{y}^{\prime}=\left(10_{1}, 0_{9}\right)$ and $\mathbf{y}^{\prime \prime}=\left(0_{1}, 0_{9}\right)$, respectively. The demand weights are understood as agents repetitions. Thus, the problem is understood as equivalent to the unweighted problem with 10 agents $(I=\{1,2, \ldots, 10\})$ where the first one corresponds to $\mathrm{C} 1$ and the further nine unweighted agents correspond to single agent $\mathrm{C} 2$. In this disaggregated form, the outcome vectors generated by two allocations $\mathrm{A} 1$ and $\mathrm{A} 2$ are given as $\mathbf{y}^{\prime}=(10,0,0,0,0,0,0,0,0,0)$ and $\mathbf{y}^{\prime \prime}=(0,0,0,0,0,0,0,0,0,0)$, respectively. Note that outcome vector $\mathbf{y}^{\prime \prime}$ with all the coordinates 0 is obviously worse than unequal vector $\mathbf{y}^{\prime}$ with one outcome 10. Actually, $\mathbf{y}^{\prime}$ Pareto dominates $\mathbf{y}^{\prime \prime}$.

\section{B. Weighted Fair Dominance}

The fair dominance for general weighted problems can be derived by their disaggregation to the unweighted ones. It can be mathematically formalized as follows. First, we introduce the right-continuous cumulative distribution function (cdf):

$$
F_{\mathbf{y}}(d)=\sum_{i \in I_{v}} \bar{v}_{i} \delta_{i}(d), \quad \delta_{i}(d)= \begin{cases}1 & \text { if } y_{v_{i}} \leq d \\ 0 & \text { otherwise }\end{cases}
$$

which for any real (outcome) value $d$ provides the measure of outcomes smaller or equal to $d$. Next, we introduce the quantile function $F_{\mathbf{y}}^{(-1)}$ as the left-continuous inverse of the cumulative distribution function $F_{\mathbf{y}}$ :

$$
F_{\mathbf{y}}^{(-1)}(\beta)=\inf \left\{\eta: F_{\mathbf{y}}(\eta) \geq \beta\right\} \text { for } 0<\beta \leq 1 \text {. }
$$

By integrating $F_{\mathbf{y}}^{(-1)}$ one gets $F_{\mathbf{y}}^{(-2)}(0)=0$ and

$$
F_{\mathbf{y}}^{(-2)}(\beta)=\int_{0}^{\beta} F_{\mathbf{y}}^{(-1)}(\alpha) d \alpha \quad \forall 0<\beta \leq 1,
$$

where $F_{\mathbf{y}}^{(-2)}(1)=\mu(\mathbf{y})$. The graph of function $F_{\mathbf{y}}^{(-2)}(\beta)$ (with respect to $\beta$ ) take the form of concave curves. It is called Absolute Lorenz Curve (ALC) [16], due to its relation to the classical Lorenz curve used in income economics as a cumulative population versus income curve to compare equity of income distributions. Indeed, the Lorenz curve may be viewed as function $L C(\xi)=\frac{1}{\mu(\mathbf{y})} \int_{0}^{\xi} F_{\mathbf{y}}^{(-1)}(\alpha) d \alpha$ thus equivalent to function $F_{\mathbf{y}}^{(-2)}(\beta)$ normalized by the distribution average. Therefore, the classical Lorenz model is focused on equity while ignoring the average result and any perfectly equal distribution of income has the diagonal line as the Lorenz curve (the same independently from the income value). Within the ALC model both equity and values of outcomes are represented. The ALC defines the relation (partial order) equivalent to the equitable dominance. Exactly, outcome vector $\mathbf{y}^{\prime}$ equitably dominates $\mathbf{y}^{\prime \prime}$, if and only if $F_{\mathbf{y}^{\prime}}^{(-2)}(\beta) \geq F_{\mathbf{y}^{\prime \prime}}^{(-2)}(\beta)$ for all $\beta \in(0,1]$ where at least one strict inequality holds. Note that for the expanded form to the unweighted outcomes, the ALC is completely defined by the values of the (cumulated) ordered outcomes. Hence, $\bar{\theta}_{i}(\mathbf{y})=m F_{\mathbf{y}}^{(-2)}(i / m)$ for $i=1, \ldots, m$, and pointwise comparison of cumulated ordered outcomes is enough to justify equitable dominance.

Finally, the impartiality of the allocation process (5) is considered in terms that two allocation schemes leading to the same distribution (cdf) of outcomes are indifferent

$$
F_{\mathbf{y}^{\prime}}=F_{\mathbf{y}^{\prime \prime}} \Rightarrow \mathbf{y}^{\prime} \cong \mathbf{y}^{\prime \prime}
$$

The principle of transfers (6) is considered for single units of demand. Although it can can be applied directly to the outcomes of importance weighted agents in the following form: if $y_{v_{i^{\prime}}}>y_{v_{i^{\prime \prime}}}$ then

$$
\mathbf{y}^{\varepsilon}=\mathbf{y}-\frac{\varepsilon}{\bar{v}_{i^{\prime}}} \mathbf{e}_{v_{i^{\prime}}}+\frac{\varepsilon}{\bar{v}_{i^{\prime \prime}}} \mathbf{e}_{v_{i^{\prime \prime}}} \succ \mathbf{y}
$$

whenever $0<\varepsilon \leq\left(y_{i^{\prime}}-y_{i^{\prime \prime}}\right) \min \left\{\bar{v}_{i^{\prime}}, \bar{v}_{i^{\prime \prime}}\right\}$ and $F_{\mathbf{y}^{\varepsilon}} \neq F_{\mathbf{y}}$.

Alternatively, the fair dominance can be expressed on the cumulative distribution functions. one may further integrate the cdf (15) to get the second order cumulative distribution function $F_{\mathbf{y}}^{(2)}(\eta)=\int_{-\infty}^{\eta} F_{\mathbf{y}}(\xi) d \xi$ for $\eta \in R$, representing average shortage to any real target $\eta$. By the theory of convex conjugate functions, the pointwise comparison of the second order cumulative distribution functions provides an alternative characterization of the equitable dominance relation [16]. Exactly, $\mathbf{y}^{\prime}$ fairly dominates $\mathbf{y}^{\prime \prime}$, if and only if $F_{\mathbf{y}^{\prime}}^{(2)}(\eta) \leq F_{\mathbf{y}^{\prime \prime}}^{(2)}(\eta)$ for all $\eta$ where at least one strict inequality holds.

Furthermore, the classical results of majorization theory [11] allow us to refer the equitable dominance to the mean utility. For any convex, increasing utility function $u: R \rightarrow R$, if outcome vector $\mathbf{y}^{\prime}$ fairly dominates $\mathbf{y}^{\prime \prime}$, then

$$
\sum_{i=1}^{m} \frac{u\left(y_{i}^{\prime}\right)}{m}=\sum_{i \in I_{v}} \bar{v}_{i} u\left(y_{v_{i}}^{\prime}\right) \geq \sum_{i=1}^{m} \frac{u\left(y_{i}^{\prime \prime}\right)}{m}=\sum_{i \in I_{v}} \bar{v}_{i} u\left(y_{v_{i}}^{\prime \prime}\right) .
$$


Finally, there are three alternative analytical characterizations of the relation of fair dominance as specified in the following theorem. Note that according to condition (iii), the fair dominance is actually the so-called increasing convex order which is more commonly known as the second degree stochastic dominance (SSD) [14].

Theorem 1: For any outcome vectors $\mathbf{y}^{\prime}, \mathbf{y}^{\prime \prime} \in A$ each of the three following conditions is equivalent to the (weak) equitable dominance $\mathbf{y}^{\prime} \succeq_{e} \mathbf{y}^{\prime \prime}$ :

(i) $F_{\mathbf{y}^{\prime}}^{(-2)}(\beta) \geq F_{\mathbf{y}^{\prime \prime}}^{(-2)}(\beta)$ for all $\beta \in(0,1]$;

(ii) $F_{\mathbf{y}^{\prime}}^{(2)}(\eta) \leq F_{\mathbf{y}^{\prime \prime}}^{(2)}(\eta)$ for all real $\eta$;

(iii) $\sum_{i \in I_{v}} \bar{v}_{i} u\left(y_{i}^{\prime}\right) \geq \sum_{i \in I_{v}} \bar{v}_{i} u\left(y_{i}^{\prime \prime}\right)$ for any concave, increasing function $u$.

Proof: Due to the finite number of agents $m$, for all vectors $\mathbf{y}$ the corresponding distributions $F_{\mathbf{y}}$ are discrete. Therefore, the equivalence of the (weak) equitable dominance $\mathbf{y}^{\prime} \succeq_{e} \mathbf{y}^{\prime \prime}$ and the ALC inequalities (i) follows as the limiting result from the duplication of agents according to their importance weights. Note that the replacement of possibly various outcomes corresponding to different copies of the same agent with their mean is clearly achievable by the equitable transfers. Alternatively, one may adapt the standard constructive proof for the unweighted case [11] to the modified axioms of the impartiality (18) and equitable transfers (19).

The equivalence of (i) and (ii) has been shown in [16] by demonstrating that for any distribution, the functions $F^{(2)}$ and $F^{(-2)}$ are convex conjugate functions.

The equivalence of (ii) and (iii) is the standard result of the stochastic orders [14].

Following Theorem 1, the importance weighted fair preference models are mathematically equivalent to the risk averse preference models for the decisions under risk, where the scenarios correspond to the agents and the importance weights define their probabilities while the agent outcomes represent realizations of a return under various scenarios.

\section{Weighted Fair Aggregations}

The classical solution concepts of mean and Max-Min are well defined for aggregated models using importance weights $v_{i}>0$. Exactly, the Max-Min solution concept is defined by maximization of the minimum outcome

$$
M(\mathbf{y})=\max _{i \in I} y_{i}=\max _{i \in I_{v}} y_{v_{i}},
$$

thus not affected by the importance weights at all. The same applies to its lexicographic regularization expressed as the MMF concept.

The solution concept of the mean outcome (10) can easily accommodate the importance weights as

$$
\mu(\mathbf{y})=\frac{1}{m} \sum_{i \in I} y_{i}=\sum_{i \in I_{v}} \bar{v}_{i} y_{v_{i}} .
$$

Similarly, for any utility function $u: R \rightarrow R$ we get

$$
\mu(u(\mathbf{y}))=\frac{1}{m} \sum_{i \in I} u\left(y_{i}\right)=\sum_{i \in I_{v}} \bar{v}_{i} u\left(y_{v_{i}}\right) .
$$

Various increasing concave utility functions $u$ can be used to define the fair aggregations (22) and the resulting fair solution concepts [15]. In particular, for the case of outcomes restricted to positive values, one may use logarithmic function thus representing the Proportional Fairness approach [7].

The OWA aggregation (14) is built for equally important outcomes where only distribution of outcome values is evaluated. For instance, considering two outcomes with the OWA weights $w_{1}=0.9$ and $w_{2}=0.1$ both symmetric outcome vectors $\mathbf{y}^{1}=(0,1)$ and $\mathbf{y}^{2}=(1,0)$ result in the same OWA aggregation $O W A_{1}=O W A_{2}=0.9 \cdot 0+0.1 \cdot 1=0.1$. Nevertheless, the importance weights of outcomes can be introduced into the OWA aggregation following the rule that the importance weights $v_{i}$ define a repetition measure within the distribution (population) of outcome values while the OWA weights $w_{i}$ are applied to averages within specific quantiles of size $1 / m$ for this distribution. For instance, introducing importance weights $v_{1}=0.75$ and $v_{2}=0.25$ we replace $\mathbf{y}^{1}=$ $(0,1)$ with the distribution taking value 0 with the repetition measure 0.75 and taking value 1 with the repetition measure 0.25 while $\mathbf{y}^{2}=(1,0)$ is replaced with the distribution taking value 1 with the repetition measure 0.75 and taking value 0 with the repetition measure 0.25 . In this specific case, the distributions may easily be equivalently interpreted in terms of four dimensional space of equally important outcomes (measure 1/4 each) where the original first outcome has been triplicated, thus $\mathbf{y}^{1}=(0,0,0,1)$ and $\mathbf{y}^{2}=(1,1,1,0)$. The OWA aggregation with weights $s_{1}=0.9$ and $s_{2}=0.1$ applied to the corresponding averages within quantiles of size $1 / 2$ results then in aggregation values $0.9 \cdot 0+0.1 \cdot(0+1) / 2=0.05$ for $\mathbf{y}^{1}$ and $0.9 \cdot(0+1) / 2+0.1 \cdot 1=0.55$ for $\mathbf{y}^{2}$, respectively. Certainly, one do not need to transform all the cases to equally important outcomes in order to calculate appropriate OWA value. Such an importance weighting OWA formula was introduced as the WOWA aggregation formally defined as follows [24]

$$
W O W A(\mathbf{y})=\sum_{i=1}^{m} \omega_{i} \theta_{i}(\mathbf{y})
$$

with

$$
\omega_{i}=\omega^{*}\left(\sum_{k=1}^{i} \bar{v}_{\tau(k)}\right)-\omega^{*}\left(\sum_{k=1}^{i-1} \bar{v}_{\tau(k)}\right)
$$

where $\omega^{*}$ is piecewise linear function interpolating points $\left(\frac{i}{m}, \sum_{k=1}^{i} w_{k}\right)$ together with $(0.0)$ and $\tau$ representing the ordering permutation for $\mathbf{y}$ (i.e. $y_{\tau(i)}=\theta_{i}(\mathbf{y})$ ). Function $w^{*}$ can be defined by its generation function

$$
g(\xi)=m w_{k} \quad \text { for }(k-1) / m<\xi \leq k / m, \quad k=1, \ldots, m
$$

with the formula $w^{*}(\alpha)=\int_{0}^{\alpha} g(\xi) d \xi$. Introducing breakpoints $\alpha_{i}=\sum_{k \leq i} v_{\tau(k)}$ and $\alpha_{0}=0$ allows us to express

$$
\omega_{i}=\int_{0}^{\alpha_{i}} g(\xi) d \xi-\int_{0}^{\alpha_{i-1}} g(\xi) d \xi=\int_{\alpha_{i-1}}^{\alpha_{i}} g(\xi) d \xi
$$


and the entire WOWA aggregation as

$$
W O W A(\mathbf{y})=\sum_{i=1}^{m} \theta_{i}(\mathbf{y}) \int_{\alpha_{i-1}}^{\alpha_{i}} g(\xi) d \xi=\int_{0}^{1} g(\xi) F_{\mathbf{y}}^{(-1)}(\xi) d \xi
$$

where $F_{\mathbf{y}}^{(-1)}$ is the inverse of the cumulative distribution function. It is a stepwise function $\bar{F}_{\mathbf{y}}^{(-1)}(\xi)=\theta_{i}(\mathbf{y})$ for $\alpha_{i-1}<\xi \leq \alpha_{i}$. Hence,

$$
W O W A(\mathbf{y})=\sum_{k=1}^{m} w_{k} m \int_{(k-1) / m}^{k / m} F_{\mathbf{y}}^{(-1)}(\xi) d \xi
$$

Note that $m \int_{(k-1) / m}^{k / m} F_{\mathbf{y}}^{(-1)}(\xi) d \xi$ represents the average within the $k$-th portion of $1 / m$ smallest outcomes, the corresponding conditional mean. Hence, the formula (25) defines WOWA aggregations with preferential weights $\mathrm{w}$ as the corresponding OWA aggregation but applied to the conditional means calculated according to the importance weights $\overline{\mathbf{v}}$ instead of the original outcomes.

Theorem 2: For any importance weights $\mathbf{v}$, the WOWA aggregation defined by decreasing preferential weights $w_{1} \geq$ $w_{2} \geq \ldots \geq w_{m}$ represents fair preferences in terms of the fair dominance, i.e.,

$$
\mathbf{y}^{\prime} \succeq_{e} \mathbf{y}^{\prime \prime} \Rightarrow W O W A\left(\mathbf{y}^{\prime}\right) \geq W O W A\left(\mathbf{y}^{\prime \prime}\right)
$$

Proof: Formula (25) may be transformed to use the lefttail averages

$$
W O W A(\mathbf{y})=\sum_{k=1}^{m} m\left(w_{k}-w_{k+1}\right) \int_{0}^{k / m} F_{\mathbf{y}}^{(-1)}(x i) d \xi
$$

where $w_{m+1}=0$. Hence.

$$
W O W A(\mathbf{y})=\sum_{k=1}^{m} w_{k}^{\prime} F_{\mathbf{y}}^{(-2)}\left(\frac{k}{m}\right)
$$

with weights

$$
\begin{aligned}
& w_{k}^{\prime}=m\left(w_{k}-w_{k+1}\right), k=1, \ldots, m-1 \\
& w_{m}^{\prime}=m w_{m}
\end{aligned}
$$

Formula (26) represents the WOWA aggregation with decreasing preferential weights as the weighted combination of the $m$ ALC values with the positive weights (27). Therefore, maximization of the WOWA aggregation with decreasing preferential weights is consisted with fair dominance and it represents the fair preferences.

The WOWA aggregation is a special case of the Choquet integral which in general may require the branch and bound algorithm for optimization [5]. However, maximization of the WOWA with increasing preferential weights, similarly to the OWA optimization, can be effectively implemented with simple auxiliary linear inequalities [18].

\section{CONCLUDING REMARKS}

This paper is devoted to fairness issues in resource allocation problems. It has been shown that the importance weights can easily be accommodated in the fair preference models leading to the corresponding fair solution concepts.
The considered fairness model is primarily well suited for the centralized resource allocation problems, like the bandwidth allocation problem [1], [4], [19], [20]. Nevertheless, the classical unweighted fairness models are used as the basis for some distributed systems managements (c.f., [22]). Many distributed systems face the problem of importance weighted agents and the analyzed weighted fairness models may help to resolve them though still requiring further research on possible implementations for specific environments.

\section{ACKNOWLEDGMENT}

The research was supported by the Polish Ministry of Science and Higher Education under grant N N516 430733.

\section{REFERENCES}

[1] Bertsekas D, Gallager R. Data Networks. Englewood Cliffs: PrenticeHall, 1987.

[2] Bouveret S, Lemaitre M. Computing leximin-optimal solutions in constraints networks. Artificial Intell 2009; 173:343-364.

[3] Brams SJ, Taylor AD. Fair Division: From Cacke Cutting to Dispute Resolution. New York: Cambridge Univ Press, 1996.

[4] Denda R, Banchs A, Effelsberg W. The fairness challenge in computer networks. LNCS 2000; 1922: 208-220.

[5] Galand L, Perny P, Spanjaard O. A branch and bound algorithm for Choquet optimization in multicriteria problems. 19th Intnl Conf MCDM, Jan 2008, Auckland.

[6] Jaffe J. Bottleneck flow control. IEEE Trans Communications 1980; 7:207-237.

[7] Kelly F, Mauloo A, Tan D. Rate control for communication networks: shadow prices, proportional fairness and stability. J Oper Res Soc 1997; 49:206-217.

[8] Kostreva MM, Ogryczak W. Linear optimization with multiple equitable criteria. RAIRO Oper Res 1999:;33:275-297.

[9] Kostreva MM, Ogryczak W, Wierzbicki A. Equitable aggregations and multiple criteria analysis, Eur J Opnl Res 2004; 158:362-367.

[10] Luss H. On equitable resource allocation problems: a lexicographic minimax approach. Oper Res 1999; 47:361-378.

[11] Marshall AW, Olkin I. Inequalities: Theory of Majorization and Its Applications. New York: Academic Press, 1979.

[12] Mo J, Walrand J. Fair end-to-end window-based congestion control. IEEE/ACM Trans on Networking 2000; 8:556-567.

[13] Moulin H. Axioms of Cooperative Decision Making. New York: Cambridge Univ Press, 1988.

[14] Müller A, Stoyan D. Comparison Methods for Stochastic Models and Risks, Chichester: Wiley, 2002.

[15] Ogryczak W. Multicriteria models for fair resource allocation. Control and Cybernetics 2007; 36:303-332.

[16] Ogryczak W, Ruszczyński A. Dual stochastic dominance and related mean-risk models. SIAM J Optimization 2002; 13:60-78.

[17] Ogryczak W, Śliwiński T. On solving linear programs with the ordered weighted averaging objective. Eur J Opnl Res 2003; 148:80-91.

[18] Ogryczak W, Śliwiński T. On optimization of the importance weighted OWA aggregation of multiple criteria. LNCS 2007; 4705:804-817.

[19] Ogryczak W, Wierzbicki A, Milewski M. A multi-criteria approach to fair and efficient bandwidth allocation. OMEGA 2008; 36:451-463.

[20] Pióro M, Medhi D. Routing, Flow and Capacity Design in Communication and Computer Networks. San Francisco: Morgan-Kaufmann, 2004.

[21] Rawls J. The Theory of Justice. Cambridge: Harvard Univ Press, 1971.

[22] Rzadca K, Trystram D., Wierzbicki A. Fair Game-Theoretic Resource Management in Dedicated Grids. CCGRID 2007; 343-350

[23] Tang A, Wang J, Low SH. Is fair allocation always inefficient. IEEE INFOCOM 2004; 35-45.

[24] Torra V. The weighted OWA operator. Int J Intell Syst 1997; 12:153166.

[25] Yager RR. On ordered weighted averaging aggregation operators in multicriteria decision making. IEEE Tr Sys Man Cyber 1988; 18:183190.

[26] Young HP. Equity in Theory and Practice. Princeton: Princeton Univ Press, 1994. 